Label-free vapor selectivity by polymer-inorganic composite photonic crystals sensors

Paola Lova, and Davide Comoretto

Citation: AIP Conference Proceedings 1981, 020097 (2018); doi: 10.1063/1.5045959

View online: https://doi.org/10.1063/1.5045959

View Table of Contents: http://aip.scitation.org/toc/apc/1981/1

Published by the American Institute of Physics 


\title{
Label-Free Vapor Selectivity by Polymer-Inorganic Composite Photonic Crystals Sensors
}

\author{
Paola Lova ${ }^{\mathrm{a}}$ and Davide Comoretto ${ }^{\mathrm{a}}$ \\ ${ }^{a}$ Dipartimento di Chimica e Chimica Industriale, Università degli Studi di Genova, via Dodecaneso 31, 16146, \\ Genova, Italy.
}

\begin{abstract}
The lack of sensors for continuous and extensive detection of vapor pollutants is a concern for health and safety. Colorimetric sensors, such as polymer distributed Bragg reflectors, could achieve this task thanks to their low cost and easy signal transduction, but are affected by low vapor permeability and lack of selectivity without chemical labels. We demonstrate label-free selective sensing of organic volatile compounds by all-polymer Bragg reflectors relying on a high free volume hybrid inorganic-polymer nanocomposite to achieve vapor permeability, and on different intercalation kinetic of organic analytes to achieve selectivity.
\end{abstract}

Keywords: Polymer photonic crystals, sensing, vapor detection, label-free selectivity.

PACS: $87.15 . V v$, 87.16.dp, 78.67.Pt

\section{INTRODUCTION}

The assessment of atmospheric volatile organic compounds (VOCs) is important to estimate health risks in industrial and urban environments. ${ }^{1}$ While quantitative assessment is commonly done by non-selective detectors that reach a detection limit of part per billions, ${ }^{2}$ qualitative analyses need long time sampling, separations and detection with mass spectroscopy, photoionization or flame ionization detectors. ${ }^{3-5}$ The lack of selectivity of portable devices and the costly and time consuming procedures needed for qualitative assessments are making new colorimetric quali-quantitative sensors interesting for environmental monitoring. These devices commonly rely on arrays of chemical targets (i.e., labels), which react selectively with different analytes. ${ }^{6-9}$ These systems are simpler than established qualitative technologies, but selectivity requires the colorimetric analysis of the arrays via chemiometry, complicating the transduction. ${ }^{6-9}$

Label-free colorimetric sensing devices make use of the intrinsic response of a medium to the analytes. In this regard, photonic crystal $(\mathrm{PhC})$ sensors are promising thanks to low fabrication costs and ease of integration in labon-a-chip devices. ${ }^{10-13} \mathrm{PhC}$ are composite materials made by media with different refractive index periodically ordered in sub-micrometric lattices. ${ }^{14-16}$ The interaction between light and these dielectric lattices generates a typical optical response, consisting in diffraction peaks detectable in reflectance mode, also called photonic band gaps (PBGs). The spectral position of these peaks depends on the characteristics of the lattice (i.e. refractive index and periodicity). $\mathrm{PhC}$ sensors rely then on the optical response induced by the intercalation of an analyte within the dielectric lattice, which affects its refractive index and periodicity. ${ }^{16}$

Among PhC, inorganic mesoporous multilayers (or distributed Bragg reflectors, DBR) have been widely reported in the literature for the detection of vapor pollutants. ${ }^{13,}{ }^{17-19}$ However, the large dielectric contrast of the materials composing these systems, combined with the and to the rigidity of the inorganic structures offer moderate spectral variation upon exposure. ${ }^{20}$ This issue can be overcome using polymer DBRs that offer relatively low dielectric contrasts, and in turn spectrally narrow PBGs, which allow the detection of very small spectral variations. Polymer structures also allow stronger responses with respect to inorganic systems due to the polymer swelling upon vapor uptake. ${ }^{12,21}$ Moreover, polymer DBRs can be grown by several simple and fast techniques such as copolymers selfassembly, ${ }^{22}$ dip-coating, ${ }^{23}$ spin-coating, ${ }^{24-25}$ or even by coextrusion at the industrial scale. ${ }^{26}$

9th International Conference on "Times of Polymers and Composites"

AIP Conf. Proc. 1981, 020097-1-020097-4; https://doi.org/10.1063/1.5045959

Published by AIP Publishing. 978-0-7354-1697-0/\$30.00 
Polymer DBRs have been successfully employed for selective detection of chemical compounds in the liquid phase, ${ }^{27-28}$ but only few studies dealt with the detection of vapors, and selectivity is still challenging. ${ }^{12,}{ }^{21}$ In a previous work, we used phase changing poly(p-phenylene oxide) as the active medium in DBR sensors demonstrating selectivity to different vapor analytes. Such selectivity was allowed by the different diffusion kinetic of the analytes within the DBR, which in turn is affected by the kinetic of polymer crystallization, the physicochemical interactions between the phase changing polymer and the analytes and, among others, the analyte polarity and Van der Waals volume. ${ }^{12}$ In this work, we investigate the label-free selectivity of polymer DBR sensors were the active sensing medium is amorphous polystyrene doped with $\mathrm{ZnO}$ nanoparticles ( $\mathrm{ZnOPS}$ ). As previously reported. the use of this nanocomposite introduces free volume into the otherwise not permeable polystyrene layers allowing analyte intercalation, sensitivity of $\sim 0.5 \mathrm{ppm}$ and lower detection limit of few ppm. ${ }^{21}$

\section{METHODS}

The DBR sensors were fabricated by alternated spin-coating of cellulose acetate (CA) solution in 4-hydroxy-4methylpentan-2-one as low index medium and polystyrene loaded with $\mathrm{ZnO}$ nanoparticles prepared and casted from toluene solution as described in previous works. ${ }^{12,21}$ All the solutions had concentration ranging between $3 \%$ and $4 \%$ wt and were casted at rotation speed of 5000 RPM on glass substrates. For all the DBR samples reflectance data were collected with homemade setups based on optical fiber using a Y-fiber probe and an Avantes AvaSpec-2048 spectrometer (200-1150 nm, resolution $1.4 \mathrm{~nm}$ ). The light source was a combined deuterium-halogen Micropak DH2000BAL. The optical response to vapor exposure of the DBR sensor was measured in a closed container saturated with the VOC analytes in room condition. The optical response was recorded using an immersion fiber probe coupled to the optical setup previously described.

\section{DISCUSSION}

Figure 1a shows a schematic of the DBRs structure. The sample is made of 21 alternated layer of CA as low refractive index medium and ZnOPS as high index one. The alternated layers generate a dielectric lattice where the refractive index is modulated along the direction perpendicular to the sample surface (Figure 1b). Light refraction and reflection occurring at any DBR interface produces a diffraction pattern, detectable in the reflectance spectrum of the samples as maxima of reflectance with intensity, width and spectral position depending on the lattice refractive indexes and periodicity. ${ }^{16}$ Figure $1 \mathrm{c}$ displays the spectrum of the CA-ZnOPS sample characterized by an intense reflectance maximum at $445 \mathrm{~nm}$ assigned to the first order PBG and by a background modulated by interference, assigned to the partial reflection occurring at the top and bottom DBR surfaces.

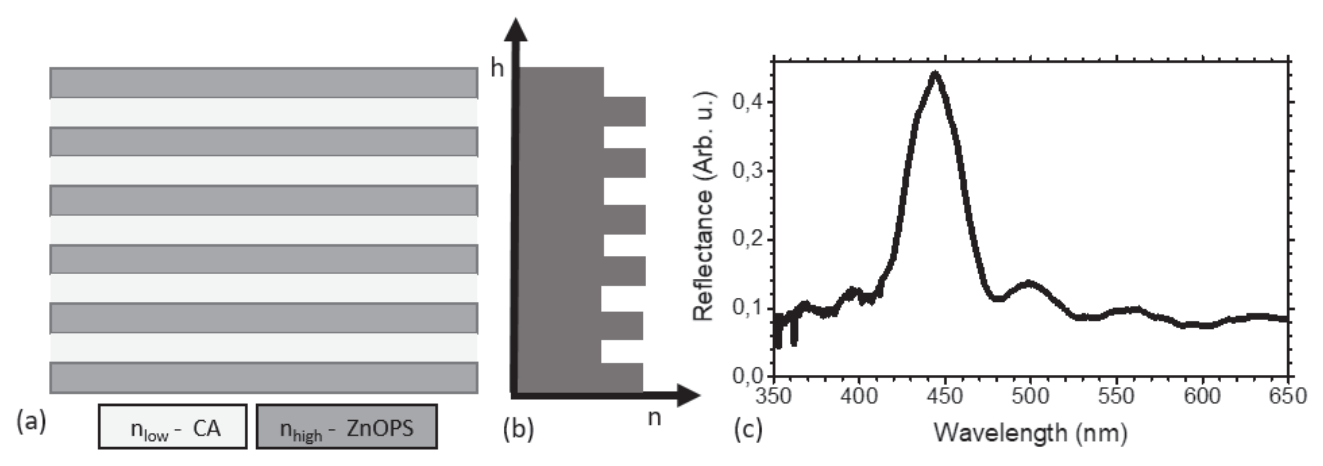

FIGURE 1. (a) Schematic of the DBR sensor structure. (b) Refractive index profile in the DBR. (c) Reflectance spectrum of a CA-DBR DBR sample.

While the sensitivity of this system was previously demonstrated, ${ }^{21}$ we exposed three portions of the same DBR to three different VOC vapors to assess selectivity. The chosen analytes were benzene, toluene, and 1,2dichlorobenzene. Figure 2 compares the reflectance spectrum of the sample collected before the exposure with the spectra collected after 10 minutes of exposure to the analytes. The DBR PBG is initially positioned at $445 \mathrm{~nm}$ (see also Figure 1c). The exposure to benzene induces a decrease in the PBG intensity and a strong red-shift to $\sim 600 \mathrm{~nm}$ 
within the exposure time (dotted line in Figure 2). This behavior has been previously associated to the intercalation of the analytes within the polymer structure and the strong swelling of the latter. ${ }^{12,21,29}$ The swelling increases the light optical path, and then the PBG shifts. When toluene is used, the optical response of the DBR sample is very different with respect to the previous one. After 10 minutes, the reflectance spectrum shows indeed a maximum at $440 \mathrm{~nm}$, which is on the short wavelength side of its initial PBG position (dashed line in Figure 2). Conversely, similarly to benzene, 1,2-dichlorobenzene exposure red-shifts the PBG position to $475 \mathrm{~nm}$.

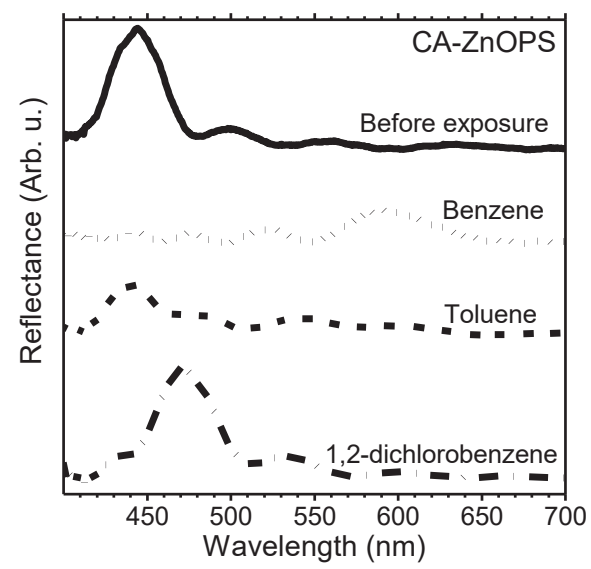

FIGURE 2: Reflectance spectra of CA-ZnOPS DBR sensors collected (from top to bottom): before exposure (continuous line) and after 10 minutes of exposure to benzene (dot lines), Toluene (dash lines) and 1,2-dichlorobenzene (dot-dash line).

In Figure 2, we notice that the shift and the intensity of the PBG detected after 10 minutes of exposure is significantly different for all the analytes, allowing their free recognition. These data agree with a progressive intercalation of the analytes within the DBR structure proceeding from the top DBR layer (closer to the environment) to the bottom one (closer to the substrate), as reported in previous works. ${ }^{12,21}$ Indeed, the analytes initially intercalate in the first layer of the DBRs and its thickness increases quickly reducing the PBG reflectance and allowing its interference with the diffraction pattern. ${ }^{12,21}$ In this case, most of the layers are unswollen and the PBG position remains unchanged, but its shape is affected from the interference resulting in an apparent shift of the maximum. This is the case of the spectra collected after 10 mins of toluene exposure (dashed line in Figure 2). When the analyte reaches the inner layers, the PBG start red-shifting due to the polymer swelling as in the case of 1,2dichlorobenzene (dash-dot line in Figure 2). When the analyte intercalates within the entire DBR, all the layers are swollen and the PBG strongly red shifts, as in the case of benzene (dot line in Figure 2). These findings are promising for new generation colorimetric sensors with broad selectivity, that do not require any complex instrumentation, and can be detected even by the naked eyes. Such sensors would enable extensive and constant monitoring of VOCs pollution in offices and households directly by the end users.

\section{CONCLUSIONS}

We demonstrated a proof-of-principle polymer inorganic composite DBR sensor able to distinguish benzene, toluene, and 1,2-dichlorobenzene. In agreement with previous findings, the different dynamic of the analyte intercalation and composite swelling allows label-free selectivity. Moreover, strong spectral variation, are detectable also by the naked eye as a color change, making these systems promising for safety devices for constant and extensive pollution monitoring in industrial and urban areas.

\section{ACKNOWLEDGMENTS}

This work was funded by the Italian Ministry of University, Research and Instruction through the "Progetti di Ricerca di Rilevante Interesse Nazionale 2010-2011" Program (Materiali Polimerici Nanostrutturati con strutture molecolari crystalline mirate, per tecnologie avanzate e per l'ambiente 2010XLLNM3) and by the European Union's Horizon 2020 research and innovation program under the Marie Sklodowska-Curie grant agreement No 643238. 


\section{REFERENCES}

1. $\quad$ Wang, P.; Zhao, W., Atmos. Res., 89, 289-297,2008.

2. $\quad$ Liu, X.; Cheng, S.; Liu, H.; Hu, S.; Zhang, D.; Ning, H., Sensors, 12, 9635-9665,2012.

3. $\quad$ Koziel, J. A.; Pawliszyn, J., J. Air Waste Manage. Assoc., 51, 173-184,2001.

4. $\quad$ Demeestere, K.; Dewulf, J.; De Witte, B.; Van Langenhove, H., J. Chromatogr. A, 1153, 130-144,2007.

5. $\quad$ Ochiai, N.; Daishima, S.; Cardin, D. B., J. Environ. Monit., 5, 997-1003,2003.

6. Wang, X.; Sun, X.; Hu, P. A.; Zhang, J.; Wang, L.; Feng, W.; Lei, S.; Yang, B.; Cao, W., Adv. Funct. Mater., 23, 6044-6050,2013.

7. $\quad$ Muro, M. L.; Daws, C. A.; Castellano, F. N., Chem. Commun., 46, 6134-6136,2008.

8. $\quad$ Eaidkong, T.; Mungkarndee, R.; Phollookin, C.; Tumcharern, G.; Sukwattanasinitt, M.; Wacharasindhu, S., J. Mater. Chem., 22, 5970-5977,2012.

9. $\quad$ Lin, H.; Jang, M.; Suslick, K. S., J. Am. Chem. Soc., 133, 16786-16789,2011.

10. Amit Kumar, G.; Hemant Sankar, D.; Suchandan, P., J. Phys. D: Appl. Phys., 50, 203001,2017.

11. Zhang, Y.; Mu, L.; Zhou, R.; Li, P.; Liu, J.; Gao, L.; Heng, L.; Jiang, L., J. Mater. Chem. C, DOI: 10.1039/C6TC03862J,2016.

12. Lova, P.; Bastianini, C.; Giusto, P.; Patrini, M.; Rizzo, P.; Guerra, G.; Iodice, M.; Soci, C.; Comoretto, D., ACS Appl. Mater. Interfaces, 8, 31941-31950,2016.

13. Lazarova, K.; Awala, H.; Thomas, S.; Vasileva, M.; Mintova, S.; Babeva, T., Sensors, 14, 12207$12218,2014$.

14. Lova, P.; Grande, V.; Manfredi, G.; Patrini, M.; Herbst, S.; Würthner, F.; Comoretto, D., Adv. Opt. Mater., 5, $1700523,2017$.

15. Manfredi, G.; Lova, P.; Di Stasio, F.; Krahne, R.; Comoretto, D., ACS Photonics, 4, 1761-1769,2017.

16. Comoretto, D., Organic and hybrid photonic crystals. Cham, Switzerland Springer: 2015.

17. Dou, Y.; Han, J.; Wang, T.; Wei, M.; Evans, D. G.; Duan, X., J. Mater. Chem., 22, 14001-14007,2012.

18. Hidalgo, N.; Calvo, M. E.; Colodrero, S.; Miguez, H., IEEE Sensors J., 10, 1206-1212,2010.

19. Hinterholzinger, F. M.; Ranft, A.; Feckl, J. M.; Ruhle, B.; Bein, T.; Lotsch, B. V., J. Mater. Chem., 22, 10356-10362,2012.

20. Bonifacio, L. D.; Ozin, G. A.; Arsenault, A. C., Small, 7, 3153-3157,2011.

21. Lova, P.; Manfredi, G.; Boarino, L.; Comite, A.; Laus, M.; Patrini, M.; Marabelli, F.; Soci, C.; Comoretto, D., ACS Photonics, 2, 537-543,2015.

22. G., A. T. K.; Nicaise, S. M.; Gadelrab, K. R.; Alexander-Katz, A.; Ross, C. A.; Berggren, K. K., Nat. Commun., 7, 10518-10527,2016.

23. Russo, M.; Campoy-Quiles, M.; Lacharmoise, P.; Ferenczi, T. A. M.; Garriga, M.; Caseri, W. R.; Stingelin, N., J. Polym. Sci., Part B: Polym. Phys. , 50, 65-74,2012.

24. Gazzo, S.; Manfredi, G.; Pötzsch, R.; Wei, Q.; Alloisio, M.; Voit, B.; Comoretto, D., J. Polym. Sci., Part B Polym. Phys., 54, 73-80,2016.

25. Manfredi, G.; Mayrhofer, C.; Kothleitner, G.; Schennach, R.; Comoretto, D., Cellulose, 23, 2853$2862,2016$.

26. Cavallo, D.; Goossens, H.; Meijer, H. E. H., Organic and Hybrid Photonic Crystals. 1st ed.; Cham, Switzerland Springer International Publishing: 2015; pp 493.

27. Noro, A.; Tomita, Y.; Shinohara, Y.; Sageshima, Y.; Walish, J. J.; Matsushita, Y.; Thomas, E. L., Macromolecules, 47, 4103-4109,2014.

28. Chan, E. P.; Walish, J. J.; Thomas, E. L.; Stafford, C. M., Adv. Mater., 23, 4702-4706,2011.

29. Lova, P.; Manfredi, G.; Boarino, L.; Laus, M.; Urbinati, G.; Losco, T.; Marabelli, F.; Caratto, V.; Ferretti, M.; Castellano, M.; Soci, C.; Comoretto, D., Physica Status Solidi C, 12, 158-162,2015. 